\title{
Chorea, Pruritus and Polycythemia: Looking for Clues
}

\author{
Vânia Rodrigues ${ }^{1}$, Carolina Lopes², Adilson Marcolino², Madalena Pinto ${ }^{2}$ \\ ${ }^{1}$ Internal Medicine Department, Centro Hospitalar de Leiria, Leiria, Portugal \\ ${ }^{2}$ Neurology Department, Centro Hospitalar de São João, Porto, Portugal
}

How to cite this article: Rodrigues V, Lopes C, Marcolino A, Pinto M. Chorea, pruritus and polycithemia: looking for clues. EJCRIM 2019;6: doi:10.12890/2019_001039.

Conflicts of Interests: The Authors declare that there are no competing interests.

This article is licensed under a Commons Attribution Non-Commercial 4.0 License

\section{ABSTRACT}

Chorea is a movement disorder usually due to vascular, hereditary, metabolic or drug- induced causes, and has rarely been reported in association with polycythemia vera (PV). Polycythemic chorea is an uncommon clinical entity that occurs more often in elderly women. PV is a treatable cause of chorea and must be considered during the diagnostic approach. We report the case of a 75-year-old woman with involuntary movements of the mouth and face with subsequent involvement of the trunk and limbs who was admitted for investigation of the chorea. The patient had the haematological attributes of PV and a positive mutation in the janus kinase 2 (JAK2) gene, and was therefore treated with hydroxyurea which led to a marked reduction in the chorea and improvement in haematological parameters. Various aetiologies of chorea must be considered in the elderly. The present case illustrates the importance of considering PV in the differential diagnosis, since its treatment leads to chorea resolution, thus avoiding serious complications.

\section{LEARNING POINTS}

- Polycythemia vera is a sporadic myeloproliferative disorder of the haematopoietic stem cells and is a treatable cause of chorea.

- Chorea is a movement disorder with various aetiologies that is difficult to diagnose.

- Prompt treatment of polycythemia vera will lead to resolution of the chorea, with aspirin and phlebotomy being recommended in lowrisk cases and hydroxyurea in high-risk cases.

\section{KEYWORDS}

Chorea, polycythemia vera, elderly

\section{CASE DESCRIPTION}

We report the case of a 75-year-old woman with history of reactive depression, hypertension and myelodysplastic syndrome under investigation at the haematology outpatient clinic. The patient was admitted in the emergency department with a 3-month history of generalized pruritus and involuntary movements of the mouth and face with subsequent involvement of the trunk and limbs. She also referred a weight loss of $16 \mathrm{~kg}$ over the previous 6 months but denied memory loss, changes in behaviour or other symptoms. The involuntary movements had been present at the last haematology appointment but had not been investigated. The patient had no prior history of stroke, peripheral vascular disease or autoimmune disease and was not being treated with neuroleptics or other chorea-inducing drugs. Her family history was remarkable for an undefined psychiatric disease that affected her father and brother.

On physical examination, the patient was normotensive and presented erythrosis of the hands and grating injuries in the trunk. Neurological examination revealed choreic movements affecting the mouth, face and four limbs (particularly exuberant in the right lower limb), mild rigidity of the limbs and deep tendon reflexes of normal intensity and symmetrical. The remainder of the neurological examination 
was unremarkable. The patient was admitted to the neurology ward for further investigation and risperidone was started, with slight improvement of chorea.

\section{METHODS AND PROCEDURES}

Laboratory studies revealed white blood cells $9,500 / \mu \mathrm{l}$, haemoglobin $17.4 \mathrm{~g} / \mathrm{dl}$, platelets $600 \times 10^{3} / \mu \mathrm{l}$, glucose $81 \mathrm{mg} / \mathrm{dl}$, calcium level of $4.7 \mathrm{mEq} / \mathrm{l}$ and low serum erythropoietin level of $<0.1 \mathrm{mU} / \mathrm{ml}$. Hepatic function, thyroid, vitamin B12, folic acid, an autoimmune study and serology and viral markers were all normal. A brain CT and MRI showed microangiopathic leucoencephalopathy. A thoracic, abdominal and pelvic CT scan was normal.

Given the laboratory results, a bone marrow biopsy was performed and revealed hypercellularity, with erythroid and megakaryocytic predominance. A genetic test for Huntington's disease was negative. However, the V617F mutation was identified in the JAK2 gene, confirming the diagnosis of polycythemia vera. After a haematologist was consulted, hydroxyurea was started, with clear improvement of chorea along with a good haematological response.

\section{DISCUSSION}

Chorea is a movement disorder characterized by excessive spontaneous movements that are abrupt, irregularly timed and randomly distributed. It is mainly due to vascular, hereditary, metabolic or drug-induced causes and poses a diagnostic challenge in elderly patients ${ }^{[1]}$. Polycythemia vera is a sporadic myeloproliferative disorder of the haematopoietic stem cells with an annual incidence of 2-10 cases per million population, and is one of the treatable causes of chorea that must be considered during the diagnostic approach ${ }^{[1-3]}$.

Neurologic manifestations of polycythemia vera are present in $50-80 \%$ of cases, and include headache, vertigo, stroke, visual disturbances and extrapyramidal syndromes, in contrast to chorea which is a rare and infrequently reported complication present in $0.5-5 \%$ of patients ${ }^{[3,4]}$. This association was first reported by Umney and Bordachzi in $1909^{[1]}$ and only a few sporadic cases have since been described in the literature. Polycythemic chorea occurs more often in women, with a female to male ratio of 2:1, usually after the age of $50^{[5]}$.

The typical clinical picture is generalized symmetrical chorea with predominant involvement of the oro-facio-lingual muscles accompanied by hypotonia and diminished reflexes; It is usually bilateral[ ${ }^{[1-4]}$.

The precise pathophysiology of this entity is still unknown, but several mechanisms have been postulated. The fundamental abnormality appears to be an excess of erythrocytes and impaired oxygen transport or hyperviscosity and its inverse relationship with cerebral blood flow. It has been reported that the decrease in cerebral blood flow to the basal ganglia and its thalami-cortical connections may compromise these structures, making them more vulnerable to metabolic factors and toxins. It has also been suggested that the sluggish cerebral blood flow can reduce levels of cerebral serotonin and catecholamines, resulting in receptor up-regulation. Also, Aminoff et al. suggested that a reasonable mechanism was dopamine receptor hypersensitivity resulting from estrogen deficit in postmenopausal women and excessive accumulation of dopamine due to platelet congestion in cerebral vessels $s^{[1,4,5]}$. However, the pathogenesis of chorea in these patients is still subject to discussion.

PV is strongly associated with a mutation in the JAK2 gene (V617F) that renders haematopoietic stem cells very sensitive to erythropoietin and other growth factors. More than $90 \%$ of patients carry a V617F JAK2 mutation, as seen in our patient.

In the present case, the brain MRI was normal except for microangiopathic leucoencephalopathy, which is in accordance with the literature, where neuroimaging studies have not shown a consistent pattern of focal lesions in the basal ganglia in patients with polycythemic chorea and were normal in the vast majority of cases $^{[1]}$.

Polycythemic chorea can be treated symptomatically with benzodiazepines and neuroleptics and prompt treatment will lead to resolution of the chorea. Aspirin and phlebotomy are recommended in low-risk cases and hydroxyurea in high-risk cases ${ }^{[1]}$, as administered in our patient.

\section{REFERENCES}

1. Kumar H, Masiowski P, Jog M. Chorea in the elderly with mutation positive polycythemia vera: a case report. Can J Neurol Sci 2009;36:370-372.

2. Venkatesan EP, Ramadoss K, Balakrishnan R, Prakash B. Essential thrombocythemia: rare cause of chorea. Ann Indian Acad Neurol 2014;17:106-107.

3. Poewe W, Djamshidian-Tehrani A. Movement disorders in systemic diseases. Neurol Clin 2015;33:269-297.

4. Midi I, Dib H, Köseoglu M, Afsar N, Günal DI. Hemichorea associated with polycythemia vera. Neurol Sci 2006;27:439-441.

5. Liu G, Chang J, Liu Z, Qiang Q, Gu C, Zhang Y, et al. Chorea disclosing a polycythemia vera. Neuropsychiatr Dis Treat 2014:10:563-565. 